\title{
DESEMPENHO MOTOR EM RECÉM-NASCIDOS PRÉ-TERMO DE ALTO RISCO
}

\section{MOTOR PERFORMANCE IN PREMATURE NEWBORN OF HIGH RISK}

\author{
Carla Marques Nicolau ${ }^{1}$ \\ Anna Paula Bastos Marques Costa ${ }^{1}$ \\ Haline Omar Hazime ${ }^{1}$ \\ Vera Lúcia Jornada Krebs ${ }^{2}$
}

Nicolau CM. et al. Desempenho motor em recém-nascidos pré-termo de alto risco. Rev Bras Cresc e Desenv Hum 2011; 21(2): 327-334.

\section{RESUMO}

Objetivo: avaliar o desempenho motor em recém-nascidos pré-termo (RNPT) com risco para o desenvolvimento motor. Método: estudo prospectivo entre junho 2007 e dezembro 2008 com RN estáveis, respiração espontânea em ar ambiente e idade corrigida até 120 dias de vida. O estudo consistiu na avaliação do desempenho motor através do Test of Infant Motor Performance (TIMP), sendo aplicado por fisioterapeuta treinado, na alta hospitalar. A análise dos resultados foi realizada através de medidas descritivas e medidas de sensibilidade, especificidade e valores preditivos positivo e negativo. Resultados: Foram estudados 69 RNs com idade gestacional (IG) média 32,61 $\pm 2,69$ semanas e peso médio $1207,00 \pm 380,14$ gramas, com predomínio do sexo masculino $(62 \%)$ e dos adequados para a idade gestacional $(66,6 \%)$. Da população estudada $56 \mathrm{RN}$ apresentaram pontuação na média, 7 apresentaram pontuação abaixo da média e 6 apresentaram escores muito abaixo da média. Os RN com pontuação dentro da média apresentaram IG média 34,44 \pm 0,59 sem, peso médio de nascimento $1355,50 \pm 294,26$ gramas e permaneceram em média $11,22 \pm 7,07$ dias em oxigênio inalatório. Os RN que obtiveram desempenho abaixo da média apresentaram IG média $32,00 \pm 2,59 \mathrm{sem}$, peso médio de nascimento $1180,00 \pm$ 334,16 gramas e permaneceram $8,20 \pm 4,24$ dias sob ventilação mecânica (VM); os RN com pontuação muito abaixo da média apresentaram IG média $28,76 \pm 2,54 \mathrm{sem}$, peso de nascimento $830,00 \pm 332,06 \mathrm{~g}$ e permaneceram em média 42,50 $\pm 18,93$ dias em VM. Verificou-se $54,5 \%$ sensibilidade, $71,4 \%$ especificidade e valores preditivos positivo e negativo de $75 \%$ e 100\%. Conclusão: Os RNPT que apresentaram pior desempenho motor foram os que permaneceram em suporte ventilatório prolongado.

Palavras-chave: desenvolvimento motor; recém-nascido; prematuro.

1 Fisioterapeutas. Serviço de Fisioterapia do Instituto da Criança - Hospital das Clínicas FMUSP, São Paulo, SP, Brasil carla.nicolau@icr.usp.br/anna.costa@icr.usp.br / haline.hazime@icr.usp.br

2 Médica. Chefe do Berçário Anexo à Maternidade. Departamento de Pediatria - Instituto da Criança Hospital das Clínicas FMUSP, São Paulo, SP, Brasil - vera.krebs@icr.usp.br

Correspondência para: Carla Marques Nicolau. Av Dr Enéas de Carvalho de Aguiar, 647 - Cerqueira Cesar, São Paulo, SP CEP 05403-000. Tel: 3069-8558 / 9624-2979. E-mail: carla.nicolau@icr.usp.br 


\begin{abstract}
Objective: to access motor performance in premature newborns with risk to motor development impairment. Method: Prospective study carried between June 2007 and December 2008 with newborn infant (NI) by spontaneous breathing in air environment and corrected age until 120 days old. The study consisted on assessment of the motor performance through the Infanty Motor Performance Test (TIMP), applied for physiotherapist on the discharge hospital. Data were presented as descriptive statistics and measures of sensibility, specifics and predictive positive and negative values. Results: We studied NI with gestational age (GA) average of $32.61 \pm 2.69$ weeks and birth weight of $1207 \pm 380.14$ grams, with predominance of the masculine sex (62\%) and the adequate for gestational age $(66,6 \%)$. The population studied ( $56 \mathrm{NI})$ presented punctuation on average, 7 presented punctuation below standard and 6 presented score below standard. The NI with punctuation on average presented GA average of $34.44 \pm 0.59$ weeks, birth weight of $1355.5 \pm 294.26$ grams and remain on the average of $11.22 \pm 7.07$ days on oxygen inhaled. The NI that acquittal below standard presented GA average of $32 \pm 2.59$ weeks, birth weight of $1180 \pm 334.16$ grams and remained $8.2 \pm 4.24$ days under mechanical ventilation (MV); the NI with punctuation was below standard presented GA average of $28.76 \pm 2.54$ weeks, birth weight of $28.76 \pm$ 2.54 grams and remained on average of $42.5 \pm 18.93$ days under mechanical ventilation. We verified $54.5 \%$ sensibility, $71.4 \%$ specifics and predictive positive and negative values of $75 \%$ and $100 \%$, respectively. Conclusion: The premature newborn infants who presented worse motor performance remained on prolonged ventilator support.
\end{abstract}

Key words: motor performance; newborn infant; premature.

\section{INTRODUÇÃO}

O avanço científico e tecnológico ocorrido nas unidades de terapia intensiva neonatais desde a década de 80 levou à queda do índice de mortalidade neonatal dos recém-nascidos de risco, principalmente os pré-termo (RNPT). Conseqüentemente, tem aumentado o interesse sobre a investigação da qualidade de vida e a morbidade desses recém-nascidos ${ }^{1,2}$.

A condição de alto risco engloba a presença de fatores biológicos e sociais que podem ocorrer no período pré, peri e/ou pós-natal, juntamente com idade gestacional e peso de nascimento proporcionando uma maior probabilidade ao recémnascido de manifestar déficits em seu desenvolvimento, podendo resultar em atraso neuropsicomotor, com alterações na aquisição de habilidades motoras, cognitivas e psicossocias ${ }^{3}$, gerando uma influência do processo neuromaturacional na aquisição de habilidades funcionais ${ }^{4}$.

Existem três grupos de indicadores de risco para déficit motor sendo eles: risco biológico onde são incluídos RN com peso de nascimento $<1500$ gramas, idade gestacional $<32$ semanas, ventilação mecânica por mais de 36 horas, hemorragia peri-intraventricular graus III e IV, anormalidades do tônus muscular, Apgar $<5$ no $5^{\circ}$ minuto e infecções congênitas; risco estabelecido que são $\mathrm{RN}$ portadores de hidrocefalia, microcefalia, anormalidades cromossômicas, anormalidades músculo-esqueléticas, miopatias congênitas e infecção por HIV; e por último os RNs incluídos no grupo de risco sócio-ambiental aonde apresentam alto risco social, abuso materno de drogas e/ou álcool e anormalidades no estado comportamental ${ }^{5,6}$.

Nos últimos 15 a 20 anos a melhoria da qualidade de assistência ao $\mathrm{RN}$ de risco não 
foi acompanhada pela queda da taxa de morbidade que se manteve praticamente constante, aumentando assim o número de crianças de risco, muitas vezes acompanhadas de graves seqüelas neurológicas ${ }^{7}$ graus III e IV, anormalidades .

Diante desta situação, é importante monitorar o desenvolvimento desses recém-nascidos, de forma a detectar precocemente possíveis desvios para intervir, prevenir ou minimizar seqüelas. Existem evidencias suficientes de que quanto mais precoce for o diagnóstico de atraso e a intervenção, menor será o impacto desses problemas na vida futura da criança $^{8-10}$.

É importante avaliar o desenvolvimento motor de uma maneira criteriosa através de instrumentos que conciliem a neuromaturação com a maturação da movimentação grosseira e todos seus fatores qualitativos. Este instrumento de avaliação deve nortear os profissionais da saúde sobre a necessidade ou não do encaminhamento do RN de risco para os serviços de intervenção precoce e devem identificar quais são os componentes motores ausentes ou incompletos a serem utilizados na estratégia de intervenção do seguimento fisioterapêutico ambulatorial visando à independência nas atividades de vida diária ${ }^{4,11,12}$.

No Brasil são escassos os estudos destinados a coletar dados acerca do desenvolvimento motor dos RNPT e a avaliar a validade de testes diagnósticos de desenvolvimento. Muitos protocolos de escalas de avaliação não avaliam somente o desenvolvimento motor, portanto, são pouco específicos para a elaboração dos objetivos nos tratamentos realizados pelos fisioterapeutas.

O teste denominado Test of Infant Motor Performance (TIMP) é um teste postural e de controle motor seletivo que foi desenvolvido para avaliar a performance motora de $\mathrm{RN}$ idade gestacional a partir de 32 semanas de nascimento até os 4 - 5 meses de idade gestacional corrigida. Este teste se propõe a documentar longitudinalmente o desempenho motor de RN que apresentam risco para o atraso nas aquisições motoras ${ }^{13}$.

Assim, o objetivo é avaliar o desempenho motor de recém-nascidos pré-termo com risco para o desenvolvimento motor.

\section{MÉTODO}

Realizou-se um ensaio clínico prospectivo e longitudinal desenvolvido no Berçário Anexo à Maternidade do Hospital das Clínicas da Faculdade de Medicina da Universidade de São Paulo (FMUSP), de junho de 2007 a dezembro de 2008. Este estudo foi aprovado pela Comissão de Pesquisa e Ética do Hospital das clínicas sob protocolo número 0812/07.

Foram incluídos os RN internados no Berçário Anexo à Maternidade do Hospital das Clínicas da Faculdade de Medicina da Universidade de São Paulo (FMUSP), que preencheram os seguintes critérios de inclusão:

- Recém-nascidos pré-termo que estavam em berço comum;

- Respiração espontânea em ar ambiente;

- $\mathrm{RN}$ com idade gestacional maior que 32 semanas.

Foram considerados como critérios de exclusão os RN:

- Portadores de síndromes genéticas;

- Portadores de mal-formações congênitas graves;

- Portadores de afecções ósteo-mio-articulares;

- Portadores de deficiências sensoriais (auditiva e visual) diagnosticadas pela avaliação da equipe multidisciplinar da unidade onde os dados serão coletados;

- Portadores de anormalidades neurológicas (hipotonia ou hipertonia e crises convulsivas). 
Foi preenchido um formulário para cada recém-nascido incluído no estudo, através da consulta de seus prontuários, sendo coletadas as seguintes informações: dados pessoais dos pais, dados da gestação e parto (número de gestações, antecedentes maternos, história pós-natal, data de nascimento, tipo de parto, intercorrências no parto), Dados neonatais (sexo, idade gestacional (em semanas), peso de nascimento (em gramas), comprimento (em centímetros), perímetro cefálico (em centímetros), perímetro torácico (em centímetros), classificação quanto ao peso $\mathrm{X}$ idade gestacional (PIG / AIG / GIG) boletim de Apgar, problemas após o nascimento), Condições de saúde atuais (data da avaliação, peso atual, idade gestacional corrigida (em semanas), diagnóstico atual.

Os recém-nascidos foram incluídos no estudo após a autorização dos pais e/ou responsável através da assinatura do Termo de Consentimento Livre e Esclarecido da própria instituição onde será realizado o estudo. O instrumento de avaliação foi aplicado no dia da alta hospitalar dos recém-nascidos estudados.

Para a avaliação do desempenho motor foi utilizado um instrumento padronizado e validado cientificamente - Test of Infant Motor Performance - TIMP, que documenta as aquisições funcionais do desenvolvimento do RN. A escala de avaliação TIMP é o único instrumento de avaliação discriminativa desenvolvido para RNPT com idade gestacional corrigida a partir de 32 semanas até 4 meses de idade corrigida.

O teste consiste de 42 itens subdivididos em: 13 itens observados, onde o examinador irá pontuar a movimentação ativa do $\mathrm{RN}$ em supino; e 29 itens elicitados, onde o examinador irá desencadear e pontuar a movimentação ativa do bebê nas posições sentado, supino, prono e em pé $^{13}$. O escore dos itens observados consiste em uma escolha dicotomizada para cada item que deve ser avaliado como observado ou não observado. Cada item observado no repertório das habilidades motoras do $\mathrm{RN}$ recebe escore 1 (um) e cada item não observado recebe escore 0 (zero). $\mathrm{O}$ escore dos itens elicitados consiste em pontuar de 0 (zero) á 5 (cinco) os movimentos desencadeados pelo examinador. Tanto os itens observados quanto os elicitados são somados e através desta somatória é obtido o escore total do teste ${ }^{13}$.

Foi utilizada uma tabela caracterizada pela idade gestacional corrigida e suas variáveis de acordo com os níveis normais, com base na amostra normativa do teste, que estão subdivididos em quatro níveis (média, abaixo da média, muito abaixo da média e muitíssimo abaixo da média) onde será dada a classificação do RN conforme seu desempenho motor. Esta tabela é parte integrante do teste utilizado $^{13}$.

Este instrumento foi aplicado em cada recém - nascido no momento da alta hospitalar conforme os critérios de inclusão e exclusão por 1 fisioterapeuta do Serviço previamente treinado, identificando-se, assim, os RN que apresentavam déficit do desempenho motor, de acordo com o instrumento escolhido. Os RN foram avaliados nas posturas sentado, supino, prono e de pé, por um período mínimo de 20 minutos e máximo de 35 minutos.

Os dados foram submetidos à análise descritiva, avaliando-se a distribuição das variáveis estudadas. Os dados nominais foram descritos em termos de porcentagens e proporções. Foram calculados os valores de sensibilidade, especificidade, valores preditivos positivo e negativo.

Para o cálculo do tamanho amostral foi considerado uma diferença de $10 \%$ entre os escores de pontuação da escala TIMP, com um $\alpha=0,05$ e um poder de teste de 0,80 . Com estes parâmetros, o n calculado foi de 60 recém-nascidos.

Os dados foram processados no software Sigma Stat (Sigma Stat Statistical Software User's Manual, 2001) ${ }^{14}$ e a planilha de dados 
foi construída no programa Microsoft Excel, 2007.

Os resultados foram considerados estatisticamente significantes quando os valores de $\mathrm{p}$ foram menores do que 0,05 .

\section{RESULTADOS E DISCUSSÃO}

Foram estudados 69 recém-nascidos prétermo, com peso de nascimento médio 1207,00 $\pm 380,14$ gramas, idade gestacional (IG) média $32,61 \pm 2,69$ semanas, sendo $62 \%$ do sexo masculino e $38 \%$ do sexo feminino, $66,6 \%$ de adequados para a idade gestacional e $33,4 \%$ de pequenos para idade gestacional (Tabela 1).

Em relação ao desempenho motor da população estudada 56 (81\%) dos RN apresen-

Tabela 1: Características da população estudada $(n=69)$

\begin{tabular}{lr}
\hline Peso de nascimento (gramas) & $1207,00 \pm 380,14$ \\
Idade gestacional (semanas) & $32,61 \pm 2,69$ \\
Sexo n (\%) & $26(38 \%)$ \\
$\quad$ Feminino & $43(62 \%)$ \\
$\quad$ Masculino & \\
Adequação nutricional n (\%) & $46(66,6 \%)$ \\
$\quad$ Adequado para idade gestacional & $23(33,4 \%)$ \\
$\quad$ Pequeno para idade gestacional & \\
\hline
\end{tabular}

Tabela 2: Desempenho motor da população estudada de acordo com a TIMP

\begin{tabular}{lc}
\hline Classificação do desempenho motor & N (\%) \\
$\begin{array}{l}\text { Desempenho motor na média para a idade } \\
\text { gestacional }\end{array}$ & $56(81 \%)$ \\
$\begin{array}{l}\text { Desempenho motor abaixo da média para a idade } \\
\text { gestacional }\end{array}$ & $7(10 \%)$ \\
$\begin{array}{l}\text { Desempenho motor muito abaixo da média para } \\
\text { a idade gestacional }\end{array}$ & $6(9 \%)$ \\
\hline
\end{tabular}

taram pontuação na média para IG, 7 (10\%) apresentaram pontuação abaixo da média para IG e $6(9 \%)$ apresentaram escores muito abaixo da média para IG (Tabela 2).

Os 56 RN com pontuação na média apresentaram peso médio de nascimento 1355,50 $\pm 294,26$ gramas, IG média $34,44 \pm 0,59$ semanas e permaneceram em média $3,00 \pm 1,29$ dias em ventilação mecânica (VM), 5,33 $\pm 1,15$ dias em CPAP nasal e 11,22 \pm 7,07 dias em oxigênio inalatório. Os RN que obtiveram desempenho abaixo da média apresentaram peso médio de nascimento $1180,00 \pm 334,16$ gramas, IG média 32,00 $\pm 2,59$ semanas e permaneceram em média 8,20 \pm 4,24 dias sob VM, $2,26 \pm 1,20$ dias em CPAP nasal e 6,33 $\pm 1,20$ dias em oxigênio inalatório. Os RN com pontuação muito abaixo da média apresentaram peso de nascimento $830,00 \pm 332,06$ gramas,

Tabela 3: Características da população estudada de acordo com o desempenho motor $(n=69)$

\begin{tabular}{lrrr}
\hline & \multicolumn{1}{c}{$\begin{array}{c}\text { Média } \\
(\mathbf{n = 5 6 )}\end{array}$} & $\begin{array}{c}\text { Abaixo da média } \\
(\mathbf{n}=\mathbf{7})\end{array}$ & $\begin{array}{c}\text { Muito abaixo da média } \\
(\mathbf{n}=\mathbf{6})\end{array}$ \\
\hline Peso de nascimento (gramas) & $1355,50 \pm 294,26$ & $1180,00 \pm 334,16$ & $830,00 \pm 332,06$ \\
Idade gestacional (semanas) & $34,44 \pm 0,59$ & $32,00 \pm 2,59$ & $28,76 \pm 2,54$ \\
Ventilação mecânica (dias) & $3,00 \pm 1,29$ & $8,20 \pm 4,24$ & $42,50 \pm 18,93$ \\
CPAP nasal ( dias) & $5,33 \pm 1,15$ & $2,26 \pm 1,20$ & $8,33 \pm 6,15$ \\
Oxigênio inalatório (dias) & $11,22 \pm 7,07$ & $6,33 \pm 3,05$ & $23,66 \pm 2,29$ \\
\hline
\end{tabular}

Tabela 4: Valores da sensibilidade, especificidade e valores preditivos positivo e negativo da escala QTIMP em recém-nascido pré-termo (RNPT) de acordo com a idade gestacional ao nascimento

\begin{tabular}{ccccc} 
& Sensibilidade & Especificidade & $\begin{array}{c}\text { Valor } \\
\text { preditivo } \\
\text { positivo } \\
\mathbf{( \% )}\end{array}$ & $\begin{array}{c}\text { Valor } \\
\text { preditivo } \\
\text { negativo } \\
\mathbf{( \% )}\end{array}$ \\
\hline RNPT & $\mathbf{( \% )}$ & $\mathbf{( \% )}$ & 75 & 100 \\
\hline
\end{tabular}


IG média $28,76 \pm 2,54$ semanas e permaneceram em média 42,50 $\pm 18,93$ dias em VM, 8,33 $\pm 6,15$ dias em CPAP nasal e 23,66 $\pm 2,29$ dias em oxigênio inalatório (Tabela 3).

Vários estudos ${ }^{16-18}$ demonstram que a internação em UTIN deve ser considerada como um dos possíveis fatores de risco para o atraso no desenvolvimento. Neste estudo, constatou-se que a internação prolongada compromete o desenvolvimento neuromotor em RNPT.

Tal situação pode ser explicada pelo fato que RN internados em UTIN são privados de estímulos sensoriais adequados, sofrendo hiperestimulação com o excesso de luzes ${ }^{19}$, de alarmes ${ }^{19}$, de ruídos intermitentes e de alta intensidade ${ }^{20}$, o excesso de manuseio, de intervenções dolorosas e contínuas interrupções do ciclo sono/vigília ${ }^{20,21}$.

Algumas pesquisas demonstram que esses $\mathrm{RN}$ chegam a ser manipulados 134 vezes em apenas 24 horas $^{20-23}$, recebendo de 50 a 150 procedimentos potencialmente dolorosos ao longo do dia ${ }^{24,25}$, e que apesar dos importantes recursos presentes em uma UTIN, os RN não estão preparados para responder de maneira organizada $^{21}$ a tantos estímulos nociceptivos, podendo vir a ter comprometimento motor.

No presente estudo, os RN que apresentaram escores abaixo ou muito abaixo da média, segundo o TIMP, foram os que permaneceram um maior período em suporte ventilatório, seja ventilação mecânica invasiva, não invasiva (CPAP nasal) ou oxigênio inalatório, em relação aos RN que apresentaram escores na média.

Dados semelhantes estão presentes na literatura ${ }^{15,26-28}$ onde se destaca que o baixo peso ao nascer e idade gestacional menores ${ }^{25,26}$, acarretam em uma maior probabilidade de alterações no desenvolvimento, principalmente nas áreas adaptativas ${ }^{24}$, motora ${ }^{24}$, psiconeurológica $^{26,28}$, audiológicas ${ }^{26,28}$, oftalmológicas ${ }^{26}$ e de linguagem ${ }^{26,27}$.

Recém-nascidos que passam por condições adversas no início de suas vidas tor- nam-se vulneráveis a desenvolver distúrbios no seu desenvolvimento ${ }^{27}$. O uso da ventilação pulmonar mecânica invasiva está associada com o déficit do desenvolvimento motor sendo relatado que, em média, $80 \%$ dos recém-nascidos ventilados no período neonatal, apresentam anormalidades no desenvolvimento ${ }^{29}$. Diante disso, diversos autores concluíram que a hospitalização neonatal maior que 5 dias tem valor preditivo para anomalias significativas no desenvolvimento, isso porque a UTIN, difere muito do ambiente intrauterino. Apesar das tentativas recentes de familiarização e humanização da UTIN, este ambiente continua sendo incapaz de alcançar níveis adequados de estimulação e interação, o que acaba tornando esta criança prematura ainda mais vulnerável a distúrbios do desenvolvimento ${ }^{29}$.

Verificou-se $54,5 \%$ sensibilidade, $71,4 \%$ especificidade e valores preditivos positivo e negativo de $75 \%$ e $100 \%$ (Tabela 4 ).

No nosso estudo, a utilização da escala TIMP apresentou boa acurácia apresentando valores de especificidade e preditivo positivo acima de $70 \%$ e valor preditivo negativo de $100 \%$, demonstrando ser uma escala confiável, apesar da baixa sensibilidade, devendo ser utilizado de rotina nas unidades neonatais pela fácil aplicação e baixo custo.

Porém, é sempre válido lembrar que os testes de avaliação devem servir como ponto de partida e não devem ser supervalorizados. Em virtude de que estes são mais úteis para o início e não para se fechar diagnóstico, pois este nunca deve ser feito por um único exame, sendo necessários diversos testes seriados, para se ter uma avaliação crítica e progressiva das alterações do desenvolvimento.

Os recém-nascidos pré-termo que apresentaram pior desempenho motor foram aqueles que permaneceram em suporte ventilatório prolongado, requerendo acompanhamento ambulatorial para garantir a aquisição das habilidades motoras. 


\section{REFERÊNCIAS}

1. Pharoah POD, Stevenson CJ, Cooke RWI, Stevenson RC. Prevalence behavior disorders in low birth weight infants. Arch Dis Child 1994; 70:272-4.

2. Stjernquist K, Svenningsen NW. Extremely low-birth-weight infants less than $901 \mathrm{~g}$ : development and behvaior after 4 years of life. Acta Paediatr 1995; 84:500-6.

3. Neto RF, Caon G, Bissani C, Silva CA, Sousa M, Silva E. Características neuropsicomotoras de crianças de alto risco neurológico atendidas em um programa de follow-up. Pediatria Moderna 2006; 2:79-85.

4. Hallal CZ, Marques NR, Braccialli LMP. Aquisição de habilidades funcionais na área de mobilidade em crianças atendidas em um programa de estimulação precoce. Rev Bras Crescimento Desenvolv Hum 2008; 18(1):27-34.

5. Katz-Salamon M, Allert K, Bergström BM, Ericsson K, Hesser U, Forssberg H. Perinatal risk factors and neuromotor behaviour during neonatal period. Acta Paediatr Supp 1997; 417:27-36.

6. Han TR, Bang MS, Lim JY, Yoon BH, Kim IO. Risk factors of cerebral palsy in preterm infants. Am J Phys Med Rehabil 2002; 81:297-303.

7. Bennett FC. Evolução do desenvolvimento. In: Avery GB, Fletcher MA, MacDonald MG. Neonatologia: fisiopatologia e tratamento do recém-nascido. $4^{\text {th }}$ ed. Rio de Janeiro: Medsi; 1999; p. 1366-18.

8. Campbell SK, Kolobe THA, Osten ET, Girolani GL \& Lemke M. Test of Infant Movement Performance, research edition. Chicago, IL: University of Illinois at Chicago, 1995.

9. Aylward GP, Conceptual issues in developmental screening and assessment.
Journal of Development and Behavior Pediatrics 1997; 18(3):340-349.

10. Halpern R, Giugliani ERJ, Victora CG, Barros FC, Horta BL. Fatores de risco para suspeita de atraso no desenvolvimento neuropsicomotor aos 12 meses de vida. Jornal de Pediatria 2000; 76(6): 421-428.

11. Piper MC, Darrah J. Motor assessment of the developing infant. Philadelphia: W.B.Saunders, 1994;210 p.

12. Jeng S, Yau KT, Chen L, Hsiao S. Alberta infant motor scale: reliability and validity when used on preterm infants in Taiwan. Phys Ther 2000; 80:168-78.

13. Campell SK. The Test of Infant Motor Peformance. Test User's Manual Version 2.0. Chicago, IL: Infant Motor Performance Scales, 2005.

14. Sigma Stat: statistical software (computer program). Version 2.0. Chicago (IL): Science;1997.

15. Rugolo LMSS. Crescimento e desenvolvimento a longo prazo do prematuro extremo. J Pediatr (RJ) 2005; 81 (1 Supl): S101-S110.

16. Figueiredo DV, Formiga CKMR, Tudella E. Aplicação de um programa de estimulação sensorial em bebês pré termo em unidade de cuidados intermediários neonatais. Temas desenvolv 2003; 12(71): 15-22.

17. Pedromônico MRM, Azevedo MFK, Benjamin I. Recém-nascidos pré-termo internados em unidade de terapia intensiva: desenvolvimento da conduta interativa no primeiro ano de vida. J pediatr (Rio J) 1998; 74(4):284-90.

18. Silva RRF. Crianças com risco de apresentar atraso do desenvolvimento e crianças com atraso estabelecido: a experiência de um ambulatório multidisciplinar. (Tese de Doutorado). São Paulo: Universidade Federal de São Paulo. Escola Paulista de Medicina; 2004. s.n p.24-32. 
19. Rech VV, Maldavsky CR. O comportamento neonatal de prematuros hospitalizados internação com suas mães. Rev Bras fisioter 2004; 8(1):75-81.

20. Aita M. Assessment of neonatal nurse behaviors that prevent overstimulation in preterm infants. Intens Crit Care Nurs 2003; 19:109-18.

21. Guinsburg R, Peres CA, Almeida MFB, Balda RCX, Berenguel RC, Tonelloto J, et al. Differences in pain expression between male and female newborn infants. Pain 2000; 85:127-33.

22. Halpern R, Giugliani ERJ, Victora CG, Barros FC, Horta BL. Risk factors forsuspicion of developmental delay at 1 months old. Rev chil pediatr 2002; 73(5):529-539.

Recebido em: 08/ago./2010 Modificado em 26/nov./2010 Aceito em 16/mar./2011 